\title{
A Non-stationary Combined Ternary 5-point Subdivision Scheme with $C^{4}$ Continuity
}

\author{
Zeze Zhang*, Hongchan Zheng, Weijie Song and Baoxing Zhang
}

\begin{abstract}
In this paper, a family of non-stationary combined ternary 5-point subdivision schemes with multiple variable parameters is proposed. The construction of the scheme is based on the generalized ternary subdivision scheme of order 4 , which is built upon refinement of a family of generalized B-splines, using the variable displacements. For such a non-stationary scheme, we study its smoothness and get that it can generate $C^{2}$ interpolating limit curves and $C^{4}$ approximating limit curves. Besides, we investigate the exponential polynomial generation/reproduction property and approximation order. It can generate/reproduce certain exponential polynomials with suitable choices of the variable parameters, and reach approximation order 5 .
\end{abstract}

\section{Introduction}

Subdivision schemes are efficient tools to design smooth curves and surfaces from a given initial polyline/polyhedral mesh and they play an important role in fields like computer graphics, wavelets and Isogeometric Analysis [2]. They are generally classified into two main categories: interpolating and approximating schemes. As a matter of fact, these two types of schemes are deeply connected. Maillot and Stam [14 presented a pushback method which is applied at each refinement step to an approximating polyline, and obtained an interpolating polyline finally. Besides in order to obtain interpolating refinement rules from approximating ones, Beccari et al. [1] multiplied the associated generating functions by a proper link polynomial. There exists a class of parameter-dependent subdivision schemes, called combined subdivision schemes, which can be regarded either as a member of the approximating one or of the interpolatory one according to the specific values assumed by the parameters, without changing the length of the subdivision masks. Levin [13] firstly proposed a combined subdivision scheme which is specially designed for the task of interpolating nets of curves. Pan et al. [17] presented a combined approximating and interpolating subdivision scheme which can generate $C^{2}$ limit curves. For

Received July 7, 2019; Accepted March 13, 2020.

Communicated by Haomin Zhou.

2010 Mathematics Subject Classification. 65D17, 26A18, 39B12.

Key words and phrases. ternary non-stationary subdivision, combined scheme, convergence, exponential polynomial generation/reproduction, approximation order.

*Corresponding author. 
other references on the combined subdivision scheme refer to 15, 18,20,23 and references therein. However, previous works are restricted to the stationary schemes which can only generate/reproduce algebraic polynomials. Zheng and Zhang 25] were the first to generalize the push-back operation to the non-stationary case and construct a non-stationary combined approximating and interpolatory subdivision scheme. Considering nice performances of combined schemes, a new non-stationary combined ternary subdivision scheme is presented here, which is obtained from the generalized ternary subdivision scheme of order 4 by using the variable displacements. For such a combined scheme, we investigate its $C^{r}$ convergence and exponential polynomial generation/reproduction property and show that it can reach $C^{4}$ smoothness and generate/reproduce exponential polynomial with suitable choices of the parameters. In the interpolatory case, the approximation order of the proposed scheme is increased by 1, compared to the scheme in [16].

The remainder of this paper is organized as follows. Basic notions and definitions are introduced in Section 2. We firstly introduce the underlying basis functions and then define the corresponding ternary generalized subdivision scheme in Section 3 . The new family of non-stationary combined ternary 5-point subdivision schemes introduced in this paper is constructed in Section 4 while its properties, including the support, smoothness, exponential polynomial generation/reproduction and approximation order, are investigated in Section 5. In Section 6, we conclude the paper with a short summary and further research work.

\section{Preliminaries}

We start by providing a few basic definitions and results about subdivision which form the basis of the rest of this paper. Given a sequence of initial control points $\boldsymbol{P}^{0}=\left\{P_{i}^{0}\right.$ : $i \in \mathbb{Z}\} \in l_{0}(\mathbb{Z})$, where $l_{0}(\mathbb{Z})$ denotes the linear space of real sequences with finite support. The set of control vertices $\boldsymbol{P}^{k+1}=\left\{P_{i}^{k+1}: i \in \mathbb{Z}\right\}$ at the $(k+1)$ st level generated by a non-stationary ternary subdivision scheme is defined by

$$
\left(\boldsymbol{P}^{k+1}\right)_{i}=\left(S_{\boldsymbol{a}^{k}} \boldsymbol{P}^{k}\right)_{i}:=\sum_{j} a_{i-3 j}^{k} P_{j}^{k}, \quad k \geq 0,
$$

where $S_{\boldsymbol{a}^{k}}$ is the $k$-level subdivision operator mapping $l_{0}(\mathbb{Z})$ to $l_{0}(\mathbb{Z})$, the sequence $\boldsymbol{a}^{k}=$ $\left\{a_{i}^{k}: i \in \mathbb{Z}\right\}$ is the $k$-level mask with finite length and we denote this subdivision scheme by $\left\{S_{\boldsymbol{a}^{k}}\right\}_{k \geq 0}$. The $k$-level symbol corresponding to the mask $\boldsymbol{a}^{k}$ is $a^{k}(z)=\sum_{i \in \mathbb{Z}} a_{i}^{k} z^{i}$.

By attaching $P_{i}^{k}$ to $i / 3^{k}$ for $i \in \mathbb{Z}, k \geq 0$, we say the scheme $\left\{S_{\boldsymbol{a}^{k}}\right\}_{k \geq 0}$ converges to a continuous function $f_{\boldsymbol{P}^{0}} \in C^{0}$, for the bounded initial control sequence $\boldsymbol{P}^{0}$, if

$$
\lim _{k \rightarrow \infty}\left\|f_{\boldsymbol{P}^{0}}\left(\frac{i}{3^{k}}\right)-P_{i}^{k}\right\|_{\infty}=0 .
$$


In this case, we say the scheme $\left\{S_{\boldsymbol{a}^{k}}\right\}_{k \geq 0}$ is $C^{0}$ convergent. If $f_{\boldsymbol{P}^{0}} \in C^{l},\left\{S_{\boldsymbol{a}^{k}}\right\}_{k \geq 0}$ is said to be $C^{l}$ convergent.

To investigate the convergence and smoothness of non-stationary ternary subdivision schemes, the following definitions and results are needed.

Definition 2.1. 10 We say two non-stationary ternary subdivision schemes $\left\{S_{a^{k}}\right\}_{k \geq 0}$ and $\left\{S_{b^{k}}\right\}_{k \geq 0}$ are asymptotically equivalent, if

$$
\sum_{k \in \mathbb{Z}_{+}}\left\|S_{a^{k}}-S_{b^{k}}\right\|_{\infty}<\infty \quad \text { with } \quad\left\|S_{a^{k}}\right\|_{\infty}=\max \left\{\sum_{j}\left|a_{i-3 j}^{k}\right|: i=0,1,2\right\} .
$$

Theorem 2.2. [10] If the non-stationary subdivision scheme $\left\{S_{a^{k}}\right\}_{k \geq 0}$ is asymptotically equivalent to a stationary subdivision scheme $S_{a}$ which is $C^{0}$ convergent and has a finitely supported mask, then the non-stationary $\left\{S_{a^{k}}\right\}_{k \geq 0}$ is also $C^{0}$ convergent.

Theorem 2.3. [10 Let a non-stationary ternary subdivision scheme $\left\{S_{a^{k}}\right\}_{k \geq 0}$ and a stationary ternary subdivision scheme $\left\{S_{a}\right\}$ be two asymptotically equivalent subdivision schemes having finite masks support. If $\left\{S_{a}\right\}$ is $C^{m}$ and

$$
\sum_{k=1}^{\infty} 3^{m k}\left\|S_{a^{k}}-S_{a}\right\|_{\infty}<\infty
$$

then the non-stationary ternary subdivision scheme $\left\{S_{a}^{k}\right\}_{k \geq 0}$ is $C^{m}$.

Apart from convergence and smoothness, the property of exponential polynomial reproduction is also important for subdivision schemes due to the ability to reproduce the function from which the data is sampled and the close relationship with approximation order. Thus we now review the definition of exponential polynomial spaces.

Definition 2.4. 21] Let $T \in \mathbb{Z}_{+}$and $\gamma=\left\{\gamma_{0}, \gamma_{1}, \ldots, \gamma_{T}\right\}$ with $\gamma_{T} \neq 0$ a finite set of real or imaginary numbers. The $T$-dimensional space of exponential polynomials $V_{T, \gamma}$ is defined by

$$
V_{T, \boldsymbol{\gamma}}:=\left\{f: \mathbb{R} \rightarrow \mathbb{C}, f \in C^{T}(\mathbb{R}): \sum_{j=0}^{T} \gamma_{j} D^{j} f=0\right\},
$$

where $D^{n}$ denotes the $n$-th order differential operator.

The exponential polynomial space $V_{T, \gamma}$ can also be characterized by the following lemma.

Lemma 2.5. 21 Let $\gamma(z)=\sum_{j=0}^{T} \gamma_{j} z^{j}$ and denote by $\left\{\theta_{l}, \tau_{l}\right\}_{l=1, \ldots, N}$ the set of zeros with multiplicity satisfying

$$
\gamma^{(r)}\left(\theta_{l}\right)=0, \quad r=0, \ldots, \tau_{l}-1, l=1, \ldots, N .
$$


Then

$$
T=\sum_{l=1}^{N} \tau_{l}, \quad V_{T, \boldsymbol{\gamma}}:=\operatorname{span}\left\{x^{r} e^{\theta_{l} x}, r=0, \ldots, \tau_{l}-1, l=1, \ldots, N\right\} .
$$

The following two theorems will be useful in discussing the generation/reproduction property of non-stationary ternary subdivision schemes.

Theorem 2.6. [4] A non-stationary ternary subdivision scheme associated with symbols $\left\{a^{k}(z)\right\}_{k \geq 0}$ generates $V_{T, \gamma}$ if

$$
\frac{d^{r} a^{k}\left(\epsilon z_{l}^{k}\right)}{d z^{r}}=0, \quad r=0, \ldots, \tau_{l}-1
$$

for $\epsilon \in\left\{e^{2 \pi i / 3}, e^{4 \pi i / 3}\right\}$ and $z_{l}^{k}:=e^{-\theta_{l} / 3^{k+1}}, l=1, \ldots, N$.

Theorem 2.7. Let $z_{l}^{k}:=e^{-\theta_{l} / 3^{k+1}}, l=1, \ldots, N$. A non-stationary ternary subdivision scheme associated with symbols $\left\{a^{k}(z)\right\}_{k \geq 0}$ reproduces $V_{T, \gamma}$ if it generates $V_{T, \gamma}$ and there exists a shift parameter $\tau$ such that for each $k \geq 0$,

$$
\frac{d^{r} a^{k}\left(z_{l}^{k}\right)}{d z^{r}}=3\left(z_{l}^{k}\right)^{2 \tau-r} q_{r}(2 \tau), \quad q_{r}(z)= \begin{cases}\prod_{j=0}^{r-1}(z-j) & \text { if } r=1, \ldots, \tau_{l}-1 \\ 1 & \text { if } r=0\end{cases}
$$

\section{Ternary generalized subdivision of order $m$}

In this section, we firstly recall the underlying generalized B-spline basis functions and then give the ternary generalized subdivision rule of order $m$.

Definition 3.1. 9 Let $T$ be a given set of uniform knots $\left\{t_{i}=i l\right\}_{i \in \mathbb{Z}}$ with interval size $l$ and $\omega$ be a given frequency parameter, where $\omega=\sqrt{\alpha}, \alpha \in \mathbb{R}$ and $\alpha \leq(\pi / l)^{2}$. Generalized B-spline basis functions of order $m, N_{i, m}(t)$ are defined by the following recurrence relation $N_{i, m}(t)=\frac{1}{l} \int_{t-l}^{t} N_{i, m-1}(s) d s$ for $m \geq 3$, where

$$
N_{0,2}(t)= \begin{cases}\frac{\omega l \sin \omega t}{2(1-\cos \omega l)}, & 0 \leq t<l \\ \frac{\omega l \sin (2 \omega l-\omega t)}{2(1-\cos \omega l)}, & l \leq t<2 l \\ 0, & \text { otherwise }\end{cases}
$$

and $N_{i, 2}(t)=N_{0,2}(t-i l)$. In $(3.1)$, the basis function can be evaluated by the L'Hospital rule about $w$, when $w=0$.

Remark 3.2. 9] Depending on the type of $\alpha$ (or $\omega$ ), the definition space of generalized B-spline basis functions is respectively as follows: 
(1) When $\alpha=0$ (i.e., $\omega=0$ ), generalized B-spline basis functions are defined over the space spanned by $\left\{1, t, t^{2}, \ldots, t^{m-1}\right\}$;

(2) When $\alpha>0$ (i.e., $\omega \in \mathbb{R}^{+}$), generalized B-spline basis functions are defined over the space spanned by $\left\{\cos \omega t, \sin \omega t, 1, t, \ldots, t^{m-3}\right\}$;

(3) When $\alpha<0$ (i.e., $\omega$ is a pure imaginary number), generalized B-spline basis functions are defined over the space spanned by $\left\{\cosh \omega t, \sinh \omega t, 1, t, \ldots, t^{m-3}\right\}$.

Remark 3.3. Let $N_{i, m}(l, t)$ denote the bases of order $m$, which are based on a set of uniform $\operatorname{knots} T:=\left\{t_{i}=i l\right\}_{i \in \mathbb{Z}}$ and supported on $[i l,(m+1) l]$. If we partition the parameter axis with interval length $l / 3$, that is taking $T^{\prime}:=\left\{t_{i}=i l / 3\right\}_{i \in \mathbb{Z}}$ as knots, we have a new set of bases with this new set of knots. Denote these new bases of order $m$ by $N_{i, m}(l / 3, t)$, following Definition 3.1 we easily have

$$
\begin{gathered}
\frac{l}{3} \int_{t-l / 3}^{t} N_{i, 2}(l / 3, s) d s=N_{i, 3}(l / 3, t), \quad \frac{l}{3} \int_{t-2 l / 3}^{t-l / 3} N_{i, 2}(l / 3, s) d s=N_{i+1,3}(l / 3, t), \\
\frac{l}{3} \int_{t-l}^{t-2 l / 3} N_{i, 2}(l / 3, s) d s=N_{i+2,3}(l / 3, t) .
\end{gathered}
$$

In the following, we derive the refinement formula of generalized B-spline curves in the ternary case according to expressing the same curve by the bases $N_{i, m}(l, t)$ and $N_{i, m}(l / 3, t)$, respectively.

Theorem 3.4. Let $p_{m}(t)$ be a generalized B-spline curve of order $m(m \geq 3)$ with interval size $l$ and control points $\left\{P_{i}\right\}_{i=1}^{n}$, that is $p_{m}(t)=\sum_{i=1}^{n} P_{i} N_{i, m}(l, t)$, where $N_{i, m}(l, t)$ are the bases defined in Definition 3.1 and $t \in[m l,(n+1) l]$. Then $p_{m}(t)$ can also be defined over the bases $N_{i, m}(l / 3, t)$ as

$$
p_{m}(t)=\sum_{i=2}^{3 n-2 m+3} P_{i}^{m} N_{i+2 m-1, m}(l / 3, t),
$$

where

$$
\left\{\begin{aligned}
P_{3 i-1}^{3} & =\frac{2 \cos \frac{\omega l}{3}}{1+2 \cos \frac{\omega l}{3}} P_{i}+\frac{1}{1+2 \cos \frac{\omega l}{3}} P_{i+1}, \\
P_{3 i}^{3} & =\frac{1}{1+2 \cos \frac{\omega l}{3}} P_{i}+\frac{2 \cos \frac{\omega l}{3}}{1+2 \cos \frac{\omega l}{3}} P_{i+1}, \\
P_{3 i+1}^{3} & =\frac{1}{\left(1+2 \cos \frac{\omega l}{3}\right)^{2}} P_{i}+\frac{4\left(\cos \frac{\omega l}{3}\right)^{2}+4 \cos \frac{\omega l}{3}-1}{\left(1+2 \cos \frac{\omega l}{3}\right)^{2}} P_{i+1}+\frac{1}{\left(1+2 \cos \frac{\omega l}{3}\right)^{2}} P_{i+2},
\end{aligned}\right.
$$

and $P_{i}^{m}=\frac{1}{3}\left(P_{i}^{m-1}+P_{i+1}^{m-1}+P_{i+2}^{m-1}\right)$ for $m>3$. 
A proof of Theorem 3.4 will be given in Section 7 , which is in analogue to the binary case in [9].

For the sake of conciseness, we denote $\cos \frac{\omega l}{3}$ by $v^{0}$ in 3.2 . Let

$$
v^{k}=\frac{1}{2}\left(e^{t / 3^{k+1}}+e^{-t / 3^{k+1}}\right)
$$

for all $k \in \mathbb{N}_{0}$ which is iteratively updated by the rule

$$
v^{k+1}=\frac{1}{2} \operatorname{Re}\left(\left(v^{k}+\sqrt{\left(v^{k}\right)^{2}-1}\right)^{1 / 3}+\left(v^{k}+\sqrt{\left(v^{k}\right)^{2}-1}\right)^{-1 / 3}\right),
$$

where $v^{0} \in(0,+\infty)$ and Re stands for the real component. As a consequence, the parameter sequence $\left\{v^{k}: k \in \mathbb{N}_{0}\right\}$ satisfies $\lim _{k \rightarrow \infty} v^{k}=1$. Following Theorem 3.4, the generalized ternary subdivision scheme $S_{m}$ of order $m(m \geq 3)$ is defined as follows.

The generalized ternary subdivision $S_{3}$ of order 3 is defined by

$$
\begin{aligned}
P_{3 i-1}^{k+1} & =\frac{2 v^{k}}{1+2 v^{k}} P_{i}^{k}+\frac{1}{1+2 v^{k}} P_{i+1}^{k}, \\
P_{3 i}^{k+1} & =\frac{1}{1+2 v^{k}} P_{i}^{k}+\frac{2 v^{k}}{1+2 v^{k}} P_{i+1}^{k}, \\
P_{3 i+1}^{k+1} & =\frac{1}{\left(1+2 v^{k}\right)^{2}} P_{i}^{k}+\frac{4\left(v^{k}\right)^{2}+4 v^{k}-1}{\left(1+2 v^{k}\right)^{2}} P_{i+1}^{k}+\frac{1}{\left(1+2 v^{k}\right)^{2}} P_{i+2}^{k},
\end{aligned}
$$

and the generalized subdivision $S_{m}$ of order $m(m>3)$ is iteratively defined by

$$
\left(S_{m} P^{k}\right)_{i}=\frac{1}{3}\left(\left(S_{m-1} P^{k}\right)_{i}+\left(S_{m-1} P^{k}\right)_{i+1}+\left(S_{m-1} P^{k}\right)_{i+2}\right) .
$$

For instance, the generalized ternary subdivision scheme $S_{4}$ of order 4 is defined by

$$
\left\{\begin{array}{l}
\bar{P}_{3 i-1}^{k+1}=\frac{4\left(v^{k}\right)^{2}+4 v^{k}+2}{3\left(1+2 v^{k}\right)^{2}} P_{i-1}^{k}+\frac{8\left(v^{k}\right)^{2}+8 v^{k}}{3\left(1+2 v^{k}\right)^{2}} P_{i}^{k}+\frac{1}{3\left(1+2 v^{k}\right)^{2}} P_{i+1}^{k}, \\
\bar{P}_{3 i}^{k+1}=\frac{2 v^{k}+2}{3\left(1+2 v^{k}\right)^{2}} P_{i-1}^{k}+\frac{12\left(v^{k}\right)^{2}+8 v^{k}-1}{3\left(1+2 v^{k}\right)^{2}} P_{i}^{k}+\frac{2 v^{k}+2}{3\left(1+2 v^{k}\right)^{2}} P_{i+1}^{k}, \\
\bar{P}_{3 i+1}^{k+1}=\frac{1}{3\left(1+2 v^{k}\right)^{2}} P_{i-1}^{k}+\frac{8\left(v^{k}\right)^{2}+8 v^{k}}{3\left(1+2 v^{k}\right)^{2}} P_{i}^{k}+\frac{4\left(v^{k}\right)^{2}+4 v^{k}+2}{3\left(1+2 v^{k}\right)^{2}} P_{i+1}^{k}
\end{array} .\right.
$$

4. A non-stationary combined ternary 5-point subdivision scheme

Based on the generalized ternary subdivision scheme of order 4 introduced in Section 3 . the main purpose of this section is to construct a new non-stationary combined ternary 5 -point subdivision scheme by the variable displacements.

From the generalized ternary subdivision scheme of order 4 in $(3.4)$, we get the matrixform

$$
\left(\begin{array}{c}
\bar{P}_{3 i-1}^{k+1} \\
\bar{P}_{3 i}^{k+1} \\
\bar{P}_{3 i+1}^{k+1}
\end{array}\right)=\left(\begin{array}{ccc}
\frac{4\left(v^{k}\right)^{2}+4 v^{k}+2}{3\left(1+2 v^{k}\right)^{2}} & \frac{8\left(v^{k}\right)^{2}+8 v^{k}}{3\left(1+2 v^{k}\right)^{2}} & \frac{1}{3\left(1+2 v^{k}\right)^{2}} \\
\frac{2 v^{k}+2}{3\left(1+2 v^{k}\right)^{2}} & \frac{12\left(v^{k}\right)^{2}+8 v^{k}-1}{3\left(1+2 v^{k}\right)^{2}} & \frac{2 v^{k}+2}{3\left(1+2 v^{k}\right)^{2}} \\
\frac{1}{3\left(1+2 v^{k}\right)^{2}} & \frac{8\left(v^{k}\right)^{2}+8 v^{k}}{3\left(1+2 v^{k}\right)^{2}} & \frac{4\left(v^{k}\right)^{2}+4 v^{k}+2}{3\left(1+2 v^{k}\right)^{2}}
\end{array}\right)\left(\begin{array}{c}
P_{i-1}^{k} \\
P_{i}^{k} \\
P_{i+1}^{k}
\end{array}\right)
$$


By adding variable parameters $\alpha^{k}, \beta^{k}, \gamma^{k}, \xi^{k}$, where $\alpha^{k}, \beta^{k}, \gamma^{k}, \xi^{k}$ are real numbers depending on $k$, the ternary combined 5 -point subdivision scheme can be written in the matrix-form

$$
\left(\begin{array}{c}
P_{3 i-1}^{k+1} \\
P_{3 i}^{k+1} \\
P_{3 i+1}^{k+1}
\end{array}\right)=\left(\begin{array}{c}
\bar{P}_{3 i-1}^{k+1} \\
\bar{P}_{3 i}^{k+1} \\
\bar{P}_{3 i+1}^{k+1}
\end{array}\right)+\left(\begin{array}{ccc}
\alpha^{k} & \beta^{k} & \gamma^{k} \\
0 & \xi^{k} & 0 \\
\gamma^{k} & \beta^{k} & \alpha^{k}
\end{array}\right)\left(\begin{array}{c}
\Delta P_{i-1}^{k} \\
\Delta P_{i}^{k} \\
\Delta P_{i+1}^{k}
\end{array}\right)
$$

where

$$
\left(\begin{array}{c}
\Delta P_{i-1}^{k} \\
\Delta P_{i}^{k} \\
\Delta P_{i+1}^{k}
\end{array}\right)=\left(\begin{array}{ccccc}
-1 & 2 & -1 & 0 & 0 \\
0 & -1 & 2 & -1 & 0 \\
0 & 0 & -1 & 2 & -1
\end{array}\right)\left(\begin{array}{c}
P_{i-2}^{k} \\
P_{i-1}^{k} \\
P_{i}^{k} \\
P_{i+1}^{k} \\
P_{i+2}^{k}
\end{array}\right)
$$

i.e., the new non-stationary combined ternary 5-point subdivision scheme is obtained as

$$
\begin{aligned}
P_{3 i-1}^{k+1}= & -\alpha^{k} P_{i-2}^{k}+\left(\frac{4\left(v^{k}\right)^{2}+4 v^{k}+2}{3\left(1+2 v^{k}\right)^{2}}+2 \alpha^{k}-\beta^{k}\right) P_{i-1}^{k} \\
& +\left(\frac{8\left(v^{k}\right)^{2}+8 v^{k}}{3\left(1+2 v^{k}\right)^{2}}-\alpha^{k}+2 \beta^{k}-\gamma^{k}\right) P_{i}^{k} \\
& +\left(\frac{1}{3\left(1+2 v^{k}\right)^{2}}-\beta^{k}+2 \gamma^{k}\right) P_{i+1}^{k}-\gamma^{k} P_{i+2}^{k}, \\
P_{3 i}^{k+1}= & \left(\frac{2 v^{k}+2}{3\left(1+2 v^{k}\right)^{2}}-\xi^{k}\right) P_{i-1}^{k}+\left(\frac{12\left(v^{k}\right)^{2}+8 v^{k}-1}{3\left(1+2 v^{k}\right)^{2}}+2 \xi^{k}\right) P_{i}^{k} \\
& +\left(\frac{2 v^{k}+2}{3\left(1+2 v^{k}\right)^{2}}-\xi^{k}\right) P_{i+1}^{k}, \\
P_{3 i+1}^{k+1}= & -\gamma^{k} P_{i-2}^{k}+\left(\frac{1}{3\left(1+2 v^{k}\right)^{2}}-\beta^{k}+2 \gamma^{k}\right) P_{i-1}^{k} \\
& +\left(\frac{8\left(v^{k}\right)^{2}+8 v^{k}}{3\left(1+2 v^{k}\right)^{2}}-\alpha^{k}+2 \beta^{k}-\gamma^{k}\right) P_{i}^{k} \\
& +\left(\frac{4\left(v^{k}\right)^{2}+4 v^{k}+2}{3\left(1+2 v^{k}\right)^{2}}+2 \alpha^{k}-\beta^{k}\right) P_{i+1}^{k}-\alpha^{k} P_{i+2}^{k},
\end{aligned}
$$

with the $k$-level symbol

$$
\begin{aligned}
& a^{k}(z)=\sum_{i=-7}^{7} a_{i}^{k} z^{i} \\
= & -\gamma^{k}\left(z^{7}+z^{-7}\right)-\alpha^{k}\left(z^{5}+z^{-5}\right)+\left(\frac{1}{3\left(1+2 v^{k}\right)^{2}}-\beta^{k}+2 \gamma^{k}\right)\left(z^{4}+z^{-4}\right) \\
& +\left(\frac{2 v^{k}+2}{3\left(1+2 v^{k}\right)^{2}}-\xi^{k}\right)\left(z^{3}+z^{-3}\right)+\left(\frac{4\left(v^{k}\right)^{2}+4 v^{k}+2}{3\left(1+2 v^{k}\right)^{2}}+2 \alpha^{k}-\beta^{k}\right)\left(z^{2}+z^{-2}\right)
\end{aligned}
$$




$$
+\left(\frac{8\left(v^{k}\right)^{2}+8 v^{k}}{3\left(1+2 v^{k}\right)^{2}}-\alpha^{k}+2 \beta^{k}-\gamma^{k}\right)\left(z+z^{-1}\right)+\frac{12\left(v^{k}\right)^{2}+8 v^{k}-1}{3\left(1+2 v^{k}\right)^{2}}+2 \xi^{k} .
$$

Remark 4.1. The non-stationary combined ternary subdivision scheme (4.1) is obtained by moving the points $\bar{P}_{3 i-1}^{k+1}, \bar{P}_{3 i}^{k+1}, \bar{P}_{3 i+1}^{k+1}$, provided by the generalized ternary subdivision scheme (3.4) of order 4 , to new points $P_{3 i-1}^{k+1}, P_{3 i}^{k+1}, P_{3 i+1}^{k+1}$, according to the variable displacements $\alpha^{k} \Delta P_{i-1}^{k}+\beta^{k} \Delta P_{i}^{k}+\gamma^{k} \Delta P_{i+1}^{k}, \xi^{k} \Delta P_{i}^{k}, \gamma^{k} \Delta P_{i-1}^{k}+\beta^{k} \Delta P_{i}^{k}+\alpha^{k} \Delta P_{i+1}^{k}$, respectively.

Remark 4.2. The new scheme (4.1) is a combined approximating and interpolatory one in the sense that it generates an approximating scheme if $\xi^{k} \neq \frac{2 v^{k}+2}{3\left(1+2 v^{k}\right)^{2}}$ and an interpolatory scheme if $\xi^{k}=\frac{2 v^{k}+2}{3\left(1+2 v^{k}\right)^{2}}$. In particular, if $\alpha^{k}=-\omega^{k}+\frac{2 v^{k}+2}{3\left(2 v^{k}-1\right)\left(1+2 v^{k}\right)^{3}}, \gamma^{k}=-\omega^{k}$, $\beta^{k}=2 v^{k} \omega^{k}\left(4\left(v^{k}\right)^{2}-3\right)+\frac{4 v^{k}\left(1+v^{k}\right)}{3\left(1+2 v^{k}\right)^{3}\left(2 v^{k}-1\right)}$, where $\omega^{k} \in \mathbb{R}$ is the free parameter, and $\xi^{k}=\frac{2 v^{k}+2}{3\left(1+2 v^{k}\right)^{3}}$, the scheme reduces to the non-stationary ternary 5-point interpolatory $C^{2}$ subdivision scheme in 16 assuming $\omega^{k}$ converges to $\omega \in(1 / 324,1 / 162)$ with the rate $O\left(3^{-2 k}\right)$ as $k \rightarrow \infty$.

Setting $\alpha^{k}=-\frac{4 v^{k}+3}{27\left(v^{k}+1\right)\left(2 v^{k}+1\right)^{2}}, \beta^{k}=-\frac{4 v^{k}\left(v^{k}+1\right)}{27\left(1+2 v^{k}\right)^{2}}, \gamma^{k}=\frac{1}{54\left(v^{k}+1\right)\left(2 v^{k}+1\right)^{2}}$ and $\xi^{k}=$ $-\frac{\left(8 v^{k}+7\right)\left(2 v^{k}-1\right)}{54\left(v^{k}+1\right)\left(2 v^{k}+1\right)}$, the non-stationary combined ternary subdivision scheme 4.1) turns into an approximating subdivision scheme

$$
\begin{aligned}
P_{3 i-1}^{k+1}= & \frac{4 v^{k}+3}{27\left(v^{k}+1\right)\left(2 v^{k}+1\right)^{2}} P_{i-2}^{k}+\frac{2\left(20\left(v^{k}\right)^{3}+40\left(v^{k}\right)^{2}+25 v^{k}+6\right)}{27\left(v^{k}+1\right)\left(1+2 v^{k}\right)^{2}} P_{i-1}^{k} \\
& +\frac{128\left(v^{k}\right)^{3}+256\left(v^{k}\right)^{2}+136 v^{k}+5}{54\left(1+v^{k}\right)\left(1+2 v^{k}\right)^{2}} P_{i}^{k} \\
& +\frac{4\left(v^{k}\right)^{3}+8\left(v^{k}\right)^{2}+13\left(v^{k}\right)+10}{27\left(v^{k}+1\right)\left(1+2 v^{k}\right)^{2}} P_{i+1}^{k}-\frac{1}{54\left(v^{k}+1\right)\left(2 v^{k}+1\right)^{2}} P_{i+2}^{k}, \\
P_{3 i}^{k+1}= & \frac{32\left(v^{k}\right)^{3}+64\left(v^{k}\right)^{2}+64 v^{k}+29}{54\left(1+v^{k}\right)\left(1+2 v^{k}\right)^{2}} P_{i-1}^{k}+\frac{76\left(v^{k}\right)^{3}+152\left(v^{k}\right)^{2}+71 v^{k}-2}{27\left(1+v^{k}\right)\left(1+2 v^{k}\right)^{2}} P_{i}^{k} \\
& +\frac{32\left(v^{k}\right)^{3}+64\left(v^{k}\right)^{2}+64 v^{k}+29}{54\left(1+v^{k}\right)\left(1+2 v^{k}\right)^{2}} P_{i+1}^{k}, \\
P_{3 i+1}^{k+1}= & -\frac{1}{54\left(v^{k}+1\right)\left(2 v^{k}+1\right)^{2}} P_{i-2}^{k}+\frac{4\left(v^{k}\right)^{3}+8\left(v^{k}\right)^{2}+13\left(v^{k}\right)+10}{27\left(v^{k}+1\right)\left(1+2 v^{k}\right)^{2}} P_{i-1}^{k} \\
& +\frac{128\left(v^{k}\right)^{3}+256\left(v^{k}\right)^{2}+136 v^{k}+5}{54\left(1+v^{k}\right)\left(1+2 v^{k}\right)^{2}} P_{i}^{k} \\
& +\frac{2\left(20\left(v^{k}\right)^{3}+40\left(v^{k}\right)^{2}+25 v^{k}+6\right)}{27\left(v^{k}+1\right)\left(1+2 v^{k}\right)^{2}} P_{i+1}^{k}+\frac{4 v^{k}+3}{27\left(v^{k}+1\right)\left(2 v^{k}+1\right)^{2}} P_{i+2}^{k} .
\end{aligned}
$$

Figure 4.1 shows the limit basic functions of the subdivision scheme 4.3 with $v^{0}=0.2$, 1 and 5. 


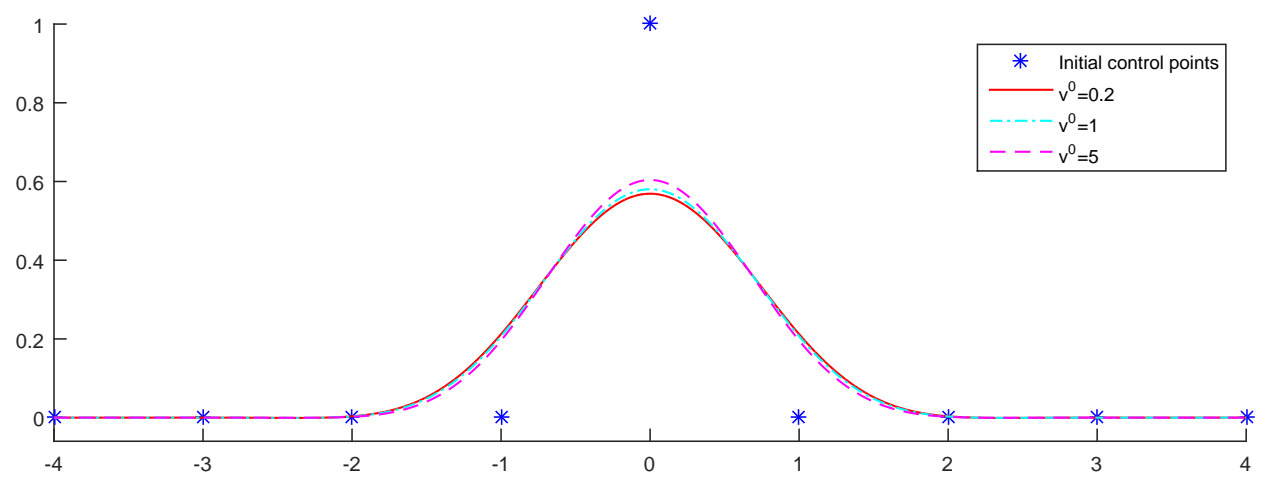

Figure 4.1: The basic limit functions of the approximating scheme 4.3.

Suppose the refinement rules in (4.1) do not depend on the level $k$, then the scheme reduces to the stationary combined one

$$
\begin{aligned}
P_{3 i-1}^{k+1}= & -\alpha P_{i-2}^{k}+\left(\frac{10}{27}+2 \alpha-\beta\right) P_{i-1}^{k}+\left(\frac{16}{27}-\alpha+2 \beta-\gamma\right) P_{i}^{k} \\
& +\left(\frac{1}{27}-\beta+2 \gamma\right) P_{i+1}^{k}-\gamma P_{i+2}^{k}, \\
P_{3 i}^{k+1}= & \left(\frac{4}{27}-\xi\right) P_{i-1}^{k}+\left(\frac{19}{27}+2 \xi\right) P_{i}^{k}+\left(\frac{4}{27}-\xi\right) P_{i+1}^{k}, \\
P_{3 i+1}^{k+1}= & -\gamma P_{i-2}^{k}+\left(\frac{1}{27}-\beta+2 \gamma\right) P_{i-1}^{k}+\left(\frac{16}{27}-\alpha+2 \beta-\gamma\right) P_{i}^{k} \\
& +\left(\frac{10}{27}+2 \alpha-\beta\right) P_{i+1}^{k}-\alpha P_{i+2}^{k}
\end{aligned}
$$

with the symbol

$$
\begin{aligned}
a(z)=\sum_{i=-7}^{7} a_{i} z^{i}= & -\gamma\left(z^{7}+z^{-7}\right)-\alpha\left(z^{5}+z^{-5}\right)+\left(\frac{1}{27}-\beta+2 \gamma\right)\left(z^{4}+z^{-4}\right) \\
& +\left(\frac{4}{27}-\xi\right)\left(z^{3}+z^{-3}\right)+\left(\frac{10}{27}+2 \alpha-\beta\right)\left(z^{2}+z^{-2}\right) \\
& +\left(\frac{16}{27}-\alpha+2 \beta-\gamma\right)\left(z+z^{-1}\right)+\frac{19}{27}+2 \xi
\end{aligned}
$$

where $\alpha, \beta, \gamma, \xi$ are real constants.

Remark 4.3. Note that the stationary scheme 4.4 is also a combined one. If $\xi \neq 4 / 27$, the scheme (4.4) is an approximating subdivision scheme. In particular, when $(\alpha, \beta, \gamma, \xi)=$ $(0,0,0,0),(0,5 / 216-a, 0,5 / 216-a),(0,5 / 54,0,4 / 27-a)$, the scheme 4.4 turns into the ternary cubic B-spline scheme, the ternary 3-point approximating subdivision scheme in [22] and the ternary 3-point approximating subdivision scheme in [19], respectively. If 
$\xi=4 / 27$, the scheme (4.4) reduces to an interpolating subdivision scheme. In particular, when $(\alpha, \beta, \gamma, \xi)=(0, \beta, 0,4 / 27),(4 / 81-a, 8 / 81+2 a,-a, 4 / 27)$, the scheme 4.4 becomes the ternary 3 -point interpolating scheme in [11] and the ternary 5-point interpolating scheme in [24], respectively.

\section{Properties of the non-stationary combined ternary 5-point subdivision scheme}

In this section, we discuss the properties of the proposed non-stationary combined ternary subdivision scheme 4.1), including the support, smoothness, exponential polynomial generation/reproduction and approximation order.

\subsection{Support}

The support of a subdivision scheme represents how far one vertex affects its neighboring points, and its size directly reflects local support property of the subdivision curve. In this subsection, we study the support of the proposed non-stationary combined ternary subdivision scheme (4.1).

For convenience, we rewrite the refinement rules of the non-stationary combined ternary subdivision scheme 4.1) as

$$
\begin{aligned}
& P_{3 i-1}^{k+1}=a_{5}^{k} P_{i-2}^{k}+a_{2}^{k} P_{i-1}^{k}+a_{-1}^{k} P_{i}^{k}+a_{-4}^{k} P_{i+1}^{k}+a_{-7}^{k} P_{i+2}^{k}, \\
& P_{3 i}^{k+1}=a_{3}^{k} P_{i-1}^{k}+a_{0}^{k} P_{i}^{k}+a_{-3}^{k} P_{i+1}^{k}, \\
& P_{3 i+1}^{k+1}=a_{7}^{k} P_{i-2}^{k}+a_{4}^{k} P_{i-1}^{k}+a_{1}^{k} P_{i}^{k}+a_{-2}^{k} P_{i+1}^{k}+a_{-5}^{k} P_{i+2}^{k},
\end{aligned}
$$

where

$$
\begin{array}{ll}
a_{-1}^{k}=a_{1}^{k}=\frac{8\left(v^{k}\right)^{2}+8 v^{k}}{3\left(1+2 v^{k}\right)^{2}}-\alpha^{k}+2 \beta^{k}-\gamma^{k}, & a_{-2}^{k}=a_{2}^{k}=\frac{4\left(v^{k}\right)^{2}+4 v^{k}+2}{3\left(1+2 v^{k}\right)^{2}}+2 \alpha^{k}-\beta^{k}, \\
a_{-3}^{k}=a_{3}^{k}=\frac{2 v^{k}+2}{3\left(1+2 v^{k}\right)^{2}}-\xi^{k}, & a_{-4}^{k}=a_{4}^{k}=\frac{1}{3\left(1+2 v^{k}\right)^{2}}-\beta^{k}+2 \gamma^{k}, \\
a_{0}^{k}=\frac{12\left(v^{k}\right)^{2}+8 v^{k}-1}{3\left(1+2 v^{k}\right)^{2}}+2 \xi^{k}, & a_{-5}^{k}=a_{5}^{k}=-\alpha^{k}, \quad a_{-7}^{k}=a_{7}^{k}=-\gamma^{k} .
\end{array}
$$

Theorem 5.1. Let $\phi_{m}:=\lim _{k \rightarrow \infty} S_{a^{m+k}} S_{a^{m+k-1}} \cdots S_{a^{m}} \delta_{0}(m \geq 0)$ be the basic limit functions generated by the proposed ternary non-stationary combined subdivision scheme 4.1. Then the length of the support is 7 , i.e., the basic limit function vanishes outside the interval $[-7 / 2,7 / 2]$.

Proof. The proof adapts the idea discussed in $3,7,10$ to the non-stationary case. In fact, assuming $[l(k), r(k)], k \geq 0$, are the supports of the $k$-level masks associated with a non-stationary subdivision scheme, the support of the limit basic function $\phi_{m}$ is proved to be included in $\left[L_{m}, R_{m}\right]=\left[\sum_{k=m}^{\infty} 3^{m-k-1} l(k), \sum_{k=m}^{\infty} 3^{m-k-1} r(k)\right]$. 
From (5.1) we have $l(k)=-7, r(k)=7$, for $k \geq 0$. Thus, for $m \geq 0$, the left endpoint

$$
L_{m}=\sum_{k=m}^{\infty} 3^{m-k-1} l(k)=-7 \times 3^{m-1} \sum_{k=m}^{\infty} 3^{-k}=-\frac{7}{2},
$$

while the right endpoint

$$
R_{m}=\sum_{k=m}^{\infty} 3^{m-k-1} r(k)=7 \times 3^{m-1} \sum_{k=m}^{\infty} 3^{-k}=\frac{7}{2},
$$

which completes the proof.

\subsection{Convergence and smoothness analysis}

Goal of this subsection is to discuss the smoothness of the non-stationary combined ternary subdivision scheme 4.1, and what choices of the parameter sequence $\left\{\alpha^{k}, \beta^{k}, \gamma^{k}, \xi^{k}\right\}$ can yield its $C^{l}$ convergence. To this aim, first we recall the following result and discuss the smoothness of the stationary subdivision scheme (4.4), which is the stationary counterpart of the non-stationary combined ternary subdivision scheme 4.1.

Theorem 5.2. [12 Let $S$ be a stationary ternary subdivision scheme with the symbol $a^{(0)}(z)$ and its $j$ th order difference scheme $S_{j}(j=1, \ldots, n+1)$ exist with symbol $a^{(j)}(z)=$ $\left(\frac{3 z}{1+z+z^{2}}\right)^{j} a^{(0)}(z)$. Suppose the symbol $a^{(l)}(z)$ satisfies

$$
\sum_{i} a_{3 i}^{(l)}=\sum_{i} a_{3 i+1}^{(l)}=\sum_{i} a_{3 i+2}^{(l)}, \quad l=0, \ldots, n .
$$

If there exists an $L \geq 1$ such that $\left\|\left(\frac{1}{3} S_{n+1}\right)^{L}\right\|_{\infty}<1$, then the scheme $S$ is $C^{n}$ convergent, where

$$
\begin{gathered}
\left\|\left(\frac{1}{3} S_{n+1}\right)^{L}\right\|_{\infty}=\max \left\{\sum_{j}\left|b_{i-3^{L} j}^{L}\right|: 0 \leq i<3^{L}\right\}, \\
b^{L}(z)=\prod_{l=0}^{L-1} b\left(z^{3^{l}}\right), \quad b(z)=\frac{1}{3} a^{(n+1)}(z) .
\end{gathered}
$$

By Theorem 5.2, we have the following result on the $C^{l}$ convergence of the scheme (4.4).

Corollary 5.3. Consider the scheme defined by (4.4). Then

(i) it generates $C^{0}$ limit curve when

$$
\begin{aligned}
&(\alpha, \beta, \gamma, \xi) \in \mathfrak{G}_{0} \\
&:=\left\{(\alpha, \beta, \gamma, \xi) \in \mathbb{R}^{4}: \max \left\{2|\gamma|+2\left|\alpha-\beta+\gamma+\frac{1}{27}\right|+\left|2 \beta-2 \alpha+\frac{7}{27}\right|,\right.\right. \\
&\left.\left.|\gamma|+\left|\beta-\gamma-\xi+\frac{1}{9}\right|+\left|\alpha-\beta+\xi+\frac{2}{9}\right|+|\alpha|\right\}<1\right\} ;
\end{aligned}
$$


(ii) it generates $C^{1}$ limit curve when

$$
\begin{aligned}
&(\alpha, \beta, \gamma, \xi) \in \mathfrak{G}_{1} \\
&:=\left\{(\alpha, \beta, \gamma, \xi) \in \mathbb{R}^{4}: \max \left\{6|\alpha+\gamma|+\left|6 \xi-6 \beta+\frac{1}{3}\right|,\right.\right. \\
&\left.\left.9|\gamma|+\left|6 \beta-3 \alpha-3 \xi+\frac{2}{9}\right|+\left|6 \alpha-3 \beta+\frac{1}{9}\right|\right\}<1\right\} ;
\end{aligned}
$$

(iii) it generates $C^{2}$ limit curve when

$$
\begin{aligned}
&(\alpha, \beta, \gamma, \xi) \in \mathfrak{G}_{2}:=\{(\alpha, \beta, \gamma, \xi) \in \mathbb{R}^{4}: \alpha=-\beta-\gamma+\xi \\
& \max \left\{18|\gamma|+\left|18 \beta+18 \gamma-12 \xi+\frac{1}{9}\right|,\right. \\
&\left.\left.3|\gamma|+3|\beta-2 \gamma-\xi|+\left|\frac{1}{9}-12 \beta-9 \gamma+9 \xi\right|\right\}<1\right\} ;
\end{aligned}
$$

(iv) it generates $C^{3}$ limit curve when

$$
\begin{aligned}
(\alpha, \beta, \gamma, \xi) \in \mathfrak{G}_{3}:=\{ & (\alpha, \beta, \gamma, \xi) \in \mathbb{R}^{4}: \alpha=2 \gamma+\frac{1}{2} \beta, \xi=3 \gamma+\frac{3}{2} \beta, \\
& \left.\max \left\{108|\gamma|+27\left|\frac{1}{2} \beta+8 \gamma\right|, 54|\gamma|+|27 \beta+270 \gamma+1|\right\}<1\right\} ;
\end{aligned}
$$

(v) it generates $C^{4}$ limit curve when

$$
\begin{aligned}
& (\alpha, \beta, \gamma, \xi) \in \mathfrak{G}_{4} \\
:= & \left\{(\alpha, \beta, \gamma, \xi) \in \mathbb{R}^{4}: 0<\gamma<\frac{1}{486}, \alpha=-\frac{1}{81}-2 \gamma, \beta=-\frac{2}{81}-8 \gamma, \xi=-9 \gamma-\frac{1}{27}\right\} .
\end{aligned}
$$

Proof. The claimed result is a straightforward consequence of Theorem 5.2 by considering $L=1$.

The following theorem provides the conditions to be satisfied such that the nonstationary combined ternary subdivision scheme defined in 4.1 indeed produces $C^{r}$ limit curves with $r=1, \ldots, 4$.

Theorem 5.4. Let $v^{k}$ be defined by (3.3) which satisfies $\left|1-v_{k}\right| \leq c 3^{-r k}(k \rightarrow \infty)$ with some constant $c>0$. If the stationary combined ternary subdivision scheme (4.4), which is the stationary counterpart of the non-stationary combined ternary subdivision scheme (4.1), is $C^{r}$ in $\mathfrak{G}_{r}$, and $\alpha^{k}, \beta^{k}, \gamma^{k}, \xi^{k}$ converge to $\alpha, \beta, \gamma$, $\xi$ with the rate $O\left(3^{-r k}\right)$ respectively as $k \rightarrow \infty$, then the non-stationary subdivision scheme (4.1) is $C^{r}$ with $r=1, \ldots, 4$.

Proof. Let $b^{k}(z)=\frac{1+z+z^{2}}{3} a^{k}(z)$. In order to prove the proposed non-stationary subdivision scheme 4.1 to be $C^{r}$, it is sufficient to show that the scheme $\left\{S_{b^{k}}\right\}_{k \geq 0}$ corresponding to $b^{k}(z)$ is $C^{r-1}$ where $r=1, \ldots, 4$. As $k$ tends to $\infty, b^{k}(z)$ tends to

$$
b(z)=-3 \gamma\left(z^{5}+z^{-7}\right)+3 \gamma\left(z^{4}+z^{-6}\right)-3 \alpha\left(z^{3}+z^{-5}\right)
$$




$$
\begin{aligned}
& +\left(3 \alpha-3 \beta+3 \gamma+\frac{1}{9}\right)\left(z^{2}+z^{-4}\right)+\left(3 \beta-3 \gamma-3 \xi+\frac{1}{3}\right)\left(z+z^{-3}\right) \\
& +\left(3 \alpha-3 \beta+3 \xi+\frac{2 v}{2 v+1}\right)\left(1+z^{-2}\right)-\left(6 \alpha-6 \beta-\frac{7}{9}\right) z^{-1}
\end{aligned}
$$

From Corollary 5.3, we know the scheme $S_{b}$ associated with $\{b(z)\}$ is $C^{r-1}$ in $\mathfrak{G}_{r}$ where $r=1, \ldots, 4$. Note that

$$
\begin{aligned}
& \left\|S_{b^{k}}-S_{b}\right\|_{\infty} \\
= & \max \left\{\sum_{j \in \mathbb{Z}_{+}}\left|b_{3 j}^{k}-b_{3 j}\right|, \sum_{j \in \mathbb{Z}_{+}}\left|b_{3 j+1}^{k}-b_{3 j+1}\right|, \sum_{j \in \mathbb{Z}_{+}}\left|b_{3 j+2}^{k}-b_{3 j+2}\right|\right\} \\
= & \max \left\{12\left|\alpha-\alpha_{k}\right|+12\left|\beta-\beta_{k}\right|+12\left|\gamma-\gamma_{k}\right|+4\left|\frac{1}{\left(1+2 v_{k}\right)^{2}}-\frac{1}{9}\right|,\right. \\
& \left.3\left|\alpha-\alpha_{k}\right|+6\left|\beta-\beta_{k}\right|+3\left|\gamma-\gamma_{k}\right|+3\left|\xi-\xi_{k}\right|+\left|\frac{1}{1+2 v_{k}}-\frac{1}{3}\right|\right\} \\
= & \max \left\{12\left|\alpha-\alpha_{k}\right|+12\left|\beta-\beta_{k}\right|+12\left|\gamma-\gamma_{k}\right|+\left|\frac{4\left(v_{k}+2\right)\left(v_{k}-1\right)}{9\left(1+2 v_{k}\right)^{2}}\right|,\right. \\
& \left.3\left|\alpha-\alpha_{k}\right|+6\left|\beta-\beta_{k}\right|+3\left|\gamma-\gamma_{k}\right|+3\left|\xi-\xi_{k}\right|+\left|\frac{2\left(v_{k}-1\right)}{3\left(1+2 v_{k}\right)}\right|\right\} .
\end{aligned}
$$

Together with $\alpha^{k}, \beta^{k}, \gamma^{k}, \xi^{k}$ converging to $\alpha, \beta, \gamma, \xi$ with the rate $O\left(3^{-k}\right)$ respectively as $k \rightarrow \infty$, we know that $\left\{S_{b^{k}}\right\}_{k \geq 0}$ is asymptotically equivalent to the stationary scheme $S_{b}$.

In order to show the non-stationary subdivision scheme $\left\{S_{b^{k}}\right\}_{k \geq 0}$ corresponding to $b^{k}(z)$ is $C^{r-1}$, in view of Theorem 2.3 we only need to prove that

$$
\sum_{k \in \mathbb{Z}_{+}} 3^{(r-1) k}\left\|S_{b^{k}}-S_{b}\right\|_{\infty} \leq \infty
$$

where $r=1, \ldots, 4$, which can be easily verified, since $(5.2)$ and $\alpha^{k}, \beta^{k}, \gamma^{k}, \xi^{k}$ converge to $\alpha, \beta, \gamma, \xi$ with the rate $O\left(3^{-r k}\right)$ respectively as $k \rightarrow \infty$, where $r=1, \ldots, 4$.

5.3. The exponential polynomial generation/reproduction property In this subsection, we discuss the exponential polynomial generation/reproduction property of the non-stationary combined ternary subdivision scheme (4.1). By Theorems 2.6 and 2.7, we can obtain a result on the exponential polynomial generation/reproduction property of the scheme 4.1.

Theorem 5.5. The non-stationary combined ternary subdivision scheme 4.1)

(i) generates the space $\operatorname{EP}_{\Gamma_{1}, \Lambda_{1}}:=\operatorname{span}\left\{x^{r}, e^{\lambda x}, r \in \Gamma_{1}=\{0,1\}, \lambda \in \Lambda_{1}=\{ \pm t\}\right\}$ if

$$
\beta^{k}=2 \alpha^{k} v^{k}+\left(4 v^{k}-8\left(v^{k}\right)^{3}\right) \gamma^{k}, \quad \xi^{k}=\left(4\left(v^{k}\right)^{2}-1\right)\left(\alpha^{k}-\gamma^{k}\right),
$$


while reproduces $\mathrm{EP}_{\Gamma_{1}, \Lambda_{1}}$ if

$$
\begin{aligned}
\alpha^{k} & =\frac{3\left(2 v^{k}-1\right)\left(2 v^{k}+1\right)^{3} \gamma^{k}+2 v^{k}+2}{3\left(2 v^{k}-1\right)\left(2 v^{k}+1\right)^{3}}, \quad \xi^{k}=\frac{2\left(v^{k}+1\right)}{3\left(2 v^{k}+1\right)^{2}}, \\
\beta^{k} & =\frac{-2 v^{k}\left[3\left(2 v^{k}-1\right)\left(4\left(v^{k}\right)^{2}-3\right)\left(2 v^{k}+1\right)^{3} \gamma^{k}-2 v^{k}-2\right]}{3\left(2 v^{k}-1\right)\left(2 v^{k}+1\right)^{3}}
\end{aligned}
$$

(ii) generates the space $\operatorname{EP}_{\Gamma_{2}, \Lambda_{1}}:=\operatorname{span}\left\{x^{r}, e^{\lambda x}, r \in \Gamma_{2}=\{0,1,2\}, \lambda \in \Lambda_{1}\right\}$ if

$$
\begin{gathered}
\alpha^{k}=-\frac{1+18 \gamma^{k} v^{k}\left(2 v^{k}+1\right)^{2}}{9\left(2 v^{k}+1\right)^{2}}, \quad \xi^{k}=-\frac{\left(2 v^{k}-1\right)\left(1+9 \gamma^{k}\left(2 v^{k}+1\right)^{3}\right)}{9\left(2 v^{k}+1\right)}, \\
\beta^{k}=-\frac{2 v^{k}\left[1+18 \gamma^{k}\left(v^{k}+1\right)\left(2 v^{k}-1\right)\left(2 v^{k}+1\right)^{2}\right]}{9\left(2 v^{k}+1\right)^{2}}
\end{gathered}
$$

while reproduces $\mathrm{EP}_{\Gamma_{2}, \Lambda_{1}}$ if

$$
\begin{aligned}
\alpha^{k} & =\frac{8\left(v^{k}\right)^{2}+12 v^{k}+1}{9\left(2 v^{k}-1\right)\left(2 v^{k}+1\right)^{4}}, & \beta^{k} & =\frac{2 v^{k}\left(8\left(v^{k}\right)^{3}+16\left(v^{k}\right)^{2}+18 v^{k}+9\right)}{9\left(2 v^{k}+1\right)^{4}}, \\
\gamma^{k} & =-\frac{4\left(v^{k}\right)^{2}+6 v^{k}+5}{9\left(2 v^{k}-1\right)\left(2 v^{k}+1\right)^{4}}, & \xi^{k} & =\frac{2\left(v^{k}+1\right)}{3\left(2 v^{k}+1\right)^{2}}
\end{aligned}
$$

(iii) generates the space $\operatorname{EP}_{\Gamma_{3}, \Lambda_{1}}:=\operatorname{span}\left\{x^{r}, e^{\lambda x}, r \in \Gamma_{3}=\{0,1,2,3\}, \lambda \in \Lambda_{1}\right\}$ if

$$
\begin{aligned}
\alpha^{k} & =-\frac{4 v^{k}+3}{27\left(v^{k}+1\right)\left(2 v^{k}+1\right)^{2}}, & \beta^{k} & =-\frac{4 v^{k}\left(v^{k}+1\right)}{27\left(1+2 v^{k}\right)^{2}}, \\
\gamma^{k} & =\frac{1}{54\left(v^{k}+1\right)\left(2 v^{k}+1\right)^{2}}, & \xi^{k} & =-\frac{\left(8 v^{k}+7\right)\left(2 v^{k}-1\right)}{54\left(v^{k}+1\right)\left(2 v^{k}+1\right)} ;
\end{aligned}
$$

(iv) generates the space $\mathrm{EP}_{\Gamma_{1}, \Lambda_{2}}:=\operatorname{span}\left\{x^{r}, e^{\lambda x}, r \in \Gamma_{1}, \lambda \in \Lambda_{2}=\{ \pm t, \pm 2 t\}\right\}$ if

$$
\begin{aligned}
\alpha^{k} & =-\frac{4\left(v^{k}\right)^{3}+4\left(v^{k}\right)^{2}-1}{3\left(v^{k}+1\right)\left(2 v^{k}-1\right)^{3}\left(2 v^{k}+1\right)^{4}}, & \beta^{k} & =-\frac{4 v^{k}\left(v^{k}+1\right)}{3\left(1+2 v^{k}\right)^{4}\left(2 v^{k}-1\right)^{2}}, \\
\gamma^{k} & =\frac{1}{6\left(v^{k}+1\right)\left(2 v^{k}+1\right)^{4}\left(2 v^{k}-1\right)^{3}}, & \xi^{k} & =-\frac{4\left(v^{k}\right)^{2}+2 v^{k}-1}{6\left(v^{k}+1\right)\left(2 v^{k}+1\right)^{2}\left(2 v^{k}-1\right)^{2}}
\end{aligned}
$$

(v) generates the space $\operatorname{EP}_{\Gamma_{1}, \Lambda_{2}, \Gamma_{1}}:=\operatorname{span}\left\{x^{r}, x^{r} e^{\lambda x}, r \in \Gamma_{1}, \lambda \in \Lambda_{2}\right\}$ if

$$
\begin{aligned}
\alpha^{k} & =-\frac{2\left(v^{k}\right)^{2}+4 v^{k}+1}{6 v^{k}\left(1+2 v^{k}\right)^{4}}, & \beta^{k} & =-\frac{4 v^{k}\left(v^{k}+1\right)}{3\left(1+2 v^{k}\right)^{4}}, \\
\gamma^{k} & =\frac{1}{12 v^{k}\left(2 v^{k}+1\right)^{4}}, & \xi^{k} & =-\frac{4\left(v^{k}\right)^{2}+4 v^{k}-3}{12 v^{k}\left(2 v^{k}+1\right)^{2}} .
\end{aligned}
$$

Proof. In view of Theorem 2.6, if the $k$ th level symbol $a^{k}(z)$ in 4.2 satisfies

$$
a^{k}(\epsilon)=D a^{k}(\epsilon)=a^{k}\left(\epsilon e^{ \pm t_{k+1}}\right)=0
$$


for $\epsilon \in\left\{e^{2 \pi i / 3}, e^{4 \pi i / 3}\right\}$, the scheme (4.1) generates the space $\operatorname{EP}_{\Gamma_{1}, \Lambda_{1}}$. By solving (5.3), we obtain

$$
\beta^{k}=2 \alpha^{k} v^{k}+\left(4 v^{k}-8\left(v^{k}\right)^{3}\right) \gamma^{k}, \quad \xi^{k}=\left(4\left(v^{k}\right)^{2}-1\right)\left(\alpha^{k}-\gamma^{k}\right)
$$

Thus in this case the scheme (4.1) generates the space $\mathrm{EP}_{\Gamma_{1}, \Lambda_{1}}$.

Similarly, from Theorem 2.7, if besides (5.4), the $k$-level symbol $a^{k}(z)$ satisfies

$$
a^{k}(1)=a^{k}\left(e^{ \pm t_{k+1}}\right)=3, \quad D a^{k}(1)=0,
$$

the scheme (4.1) reproduces the space $\mathrm{EP}_{\Gamma_{1}, \Lambda_{1}}$. Thus by solving (5.4) and (5.5), we get that the non-stationary combined ternary subdivision scheme (4.1) reproduces the space $\mathrm{EP}_{\Gamma_{1}, \Lambda_{1}}$ if

$$
\begin{aligned}
\alpha^{k} & =\frac{3\left(2 v^{k}-1\right)\left(2 v^{k}+1\right)^{3} \gamma^{k}+2 v^{k}+2}{3\left(2 v^{k}-1\right)\left(2 v^{k}+1\right)^{3}}, \quad \xi^{k}=\frac{2\left(v^{k}+1\right)}{3\left(2 v^{k}+1\right)^{2}}, \\
\beta^{k} & =\frac{-2 v^{k}\left[3\left(2 v^{k}-1\right)\left(4\left(v^{k}\right)^{2}-3\right)\left(2 v^{k}+1\right)^{3} \gamma^{k}-2 v^{k}-2\right]}{3\left(2 v^{k}-1\right)\left(2 v^{k}+1\right)^{3}} .
\end{aligned}
$$

In analogue to the above process to guarantee the generation/reproduction of the space $\mathrm{EP}_{\Gamma_{1}, \Lambda_{1}}$, we can derive conditions on the multiple variable parameters $\alpha^{k}, \beta^{k}, \gamma^{k}$, $\xi^{k}$, which guarantee the generation of the spaces $\mathrm{EP}_{\Gamma_{2}, \Lambda_{1}}, \mathrm{EP}_{\Gamma_{3}, \Lambda_{1}}, \mathrm{EP}_{\Gamma_{1}, \Lambda_{2}}, \mathrm{EP}_{\Gamma_{1}, \Lambda_{2}, \Gamma_{1}}$ and the reproduction of the space $\mathrm{EP}_{\Gamma_{2}, \Lambda_{1}}$, by the non-stationary combined ternary subdivision scheme (4.1) following Theorems 2.6 and 2.7 .

Remark 5.6. Note that when reproducing the space $\mathrm{EP}_{\Gamma_{2}, \Lambda_{1}}$, the non-stationary combined ternary subdivision scheme (4.1) reduces to an interpolating 5-point ternary subdivision scheme which is $C^{2}$. When generating the spaces $\mathrm{EP}_{\Gamma_{3}, \Lambda_{1}}, \mathrm{EP}_{\Gamma_{1}, \Lambda_{2}}$ and $\mathrm{EP}_{\Gamma_{1}, \Lambda_{2}, \Gamma_{1}}$ respectively, the non-stationary combined ternary subdivision scheme 4.1 turns into a $C^{4}$ approximating 5-point ternary subdivision scheme.

Setting

$$
\begin{aligned}
\left(\alpha^{k}, \beta^{k}, \gamma^{k}, \xi^{k}\right)= & \left(\frac{8\left(v^{k}\right)^{2}+12 v^{k}+1}{9\left(2 v^{k}-1\right)\left(2 v^{k}+1\right)^{4}}, \frac{2 v^{k}\left(8\left(v^{k}\right)^{3}+16\left(v^{k}\right)^{2}+18 v^{k}+9\right)}{9\left(2 v^{k}+1\right)^{4}},\right. \\
& \left.-\frac{4\left(v^{k}\right)^{2}+6 v^{k}+5}{9\left(2 v^{k}-1\right)\left(2 v^{k}+1\right)^{4}}, \frac{2\left(1+v^{k}\right)}{3\left(1+2 v^{k}\right)^{2}}\right)
\end{aligned}
$$

and

$$
\left(-\frac{4 v^{k}+3}{27\left(v^{k}+1\right)\left(2 v^{k}+1\right)^{2}},-\frac{4 v^{k}\left(v^{k}+1\right)}{27\left(1+2 v^{k}\right)^{2}}, \frac{1}{54\left(v^{k}+1\right)\left(2 v^{k}+1\right)^{2}},-\frac{\left(8 v^{k}+7\right)\left(2 v^{k}-1\right)}{54\left(v^{k}+1\right)\left(2 v^{k}+1\right)}\right),
$$


the non-stationary combined ternary subdivision scheme 4.1) turns into an interpolating scheme

$$
\begin{aligned}
P_{3 i-1}^{k+1}= & -\frac{8\left(v^{k}\right)^{2}+12 v^{k}+1}{9\left(2 v^{k}-1\right)\left(2 v^{k}+1\right)^{4}} P_{i-2}^{k} \\
& +\frac{2\left(32\left(v^{k}\right)^{5}+48\left(v^{k}\right)^{4}+16\left(v^{k}\right)^{3}+2\left(v^{k}\right)^{2}+9 v^{k}-2\right)}{9\left(2 v^{k}-1\right)\left(1+2 v^{k}\right)^{4}} P_{i-1}^{k} \\
& +\frac{2\left(64\left(v^{k}\right)^{4}+128\left(v^{k}\right)^{3}+96\left(v^{k}\right)^{2}+29 v^{k}-2\right)}{9\left(1+2 v^{k}\right)^{4}} P_{i}^{k} \\
& -\frac{32\left(v^{k}\right)^{5}+48\left(v^{k}\right)^{4}+16\left(v^{k}\right)^{3}-4\left(v^{k}\right)^{2}+13}{9\left(2 v^{k}-1\right)\left(1+2 v^{k}\right)^{4}} P_{i+1}^{k} \\
& +\frac{4\left(v^{k}\right)^{2}+6 v^{k}+5}{9\left(2 v^{k}-1\right)\left(2 v^{k}+1\right)^{4}} P_{i+2}^{k}, \\
P_{3 i}^{k+1}= & P_{i}^{k}, \\
P_{3 i+1}^{k+1}= & \frac{4\left(v^{k}\right)^{2}+6 v^{k}+5}{9\left(2 v^{k}-1\right)\left(2 v^{k}+1\right)^{4}} P_{i-2}^{k} \\
& -\frac{32\left(v^{k}\right)^{5}+48\left(v^{k}\right)^{4}+16\left(v^{k}\right)^{3}-4\left(v^{k}\right)^{2}+13}{9\left(2 v^{k}-1\right)\left(1+2 v^{k}\right)^{4}} P_{i-1}^{k} \\
& +\frac{2\left(64\left(v^{k}\right)^{4}+128\left(v^{k}\right)^{3}+96\left(v^{k}\right)^{2}+29 v^{k}-2\right)}{9\left(1+2 v^{k}\right)^{4}} P_{i}^{k} \\
& +\frac{2\left(32\left(v^{k}\right)^{5}+48\left(v^{k}\right)^{4}+16\left(v^{k}\right)^{3}+2\left(v^{k}\right)^{2}+9 v^{k}-2\right)}{9\left(2 v^{k}-1\right)\left(1+2 v^{k}\right)^{4}} P_{i+1}^{k} \\
& -\frac{8\left(v^{k}\right)^{2}+12 v^{k}+1}{9\left(2 v^{k}-1\right)\left(2 v^{k}+1\right)^{4}} P_{i+2}^{k}, \\
&
\end{aligned}
$$

which generates $C^{2}$ limit curves, and an approximating scheme 4.3 which is $C^{4}$, respectively. We compare these schemes with the interpolating 5-point ternary subdivision scheme defined in 16 which generate $C^{2}$ limit curves.

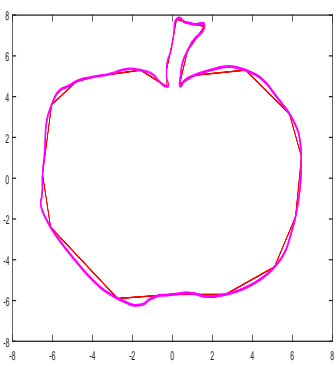

(a) $C^{2}$ continuity

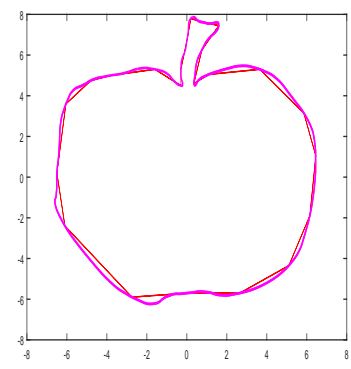

(b) $C^{2}$ continuity

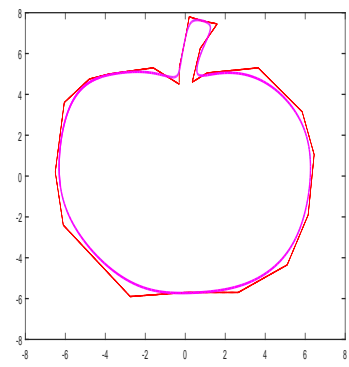

(c) $C^{4}$ continuity

Figure 5.1: Different limit curves for Apple generated by the scheme introduced in [16], the schemes (5.6) and (4.3) with $v^{0}=0.8$ (from left to right). 
Figure 5.1 illustrates the limit curves, which have the same control polygon, generated by these schemes with $v^{0}=0.8$. Figure 5.2 displays the reproduction of the circle (left), hyperbola (center) and quartic function (right) with $v^{0}=\cos \frac{\pi}{3}, \cosh \frac{2}{3}$ and 1 by the interpolating scheme (5.6), respectively.
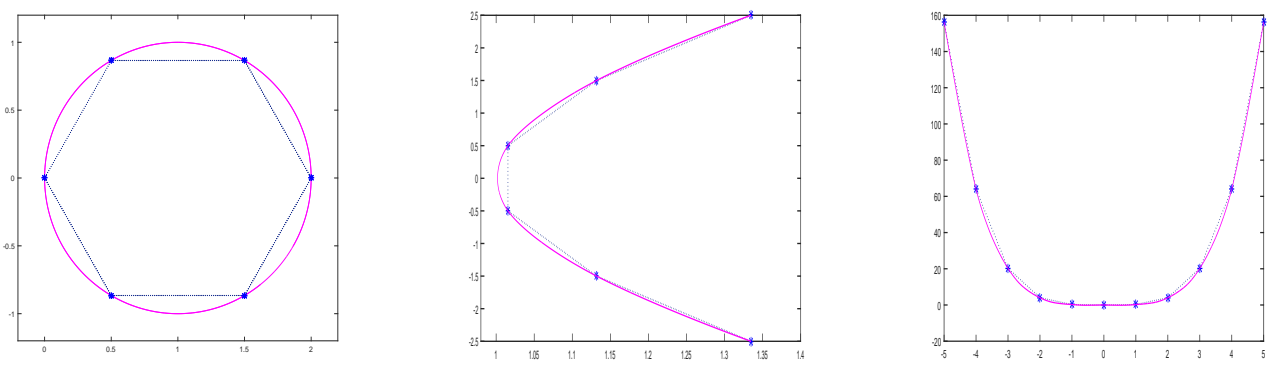

Figure 5.2: Limit curves generated by the scheme (5.6) with $v^{0}=\cos \frac{\pi}{3}, \cosh \frac{2}{3}, 1$ (from left to right).

\subsection{The approximation order}

The approximation order has the close relationship with the property of exponential reproduction. Based on Subsection 5.3, we investigate the approximation order of proposed non-stationary combined ternary subdivision scheme (4.1) in this subsection. For simplicity, in the following we rewrite $T$-dimensional space of exponential polynomials $V_{T, \gamma}$ introduced in Definition 2.4 as $V_{T}:=\operatorname{span}\left\{\varphi_{0}(x), \ldots, \varphi_{T-1}(x)\right\}$, and $W_{\infty}^{n}(\mathbb{R}), n \in \mathbb{N}$ denotes the Sobolev space.

Definition 5.7. 5] Suppose that $\left\{S_{\boldsymbol{a}^{k}}\right\}_{k \geq 0}$ is a non-stationary ternary scheme and $f^{0}=$ $\left\{f_{i}^{0}: i \in \mathbb{Z}\right\}$ is sampled from an underlying function $f$ with density $3^{-k_{0}}$ for some $k_{0} \in \mathbb{Z}$. The largest exponent $d>0$ such that

$$
\left\|S_{a^{k}}^{\infty} f^{0}-f\right\|_{L_{\infty}(\mathbb{K})} \leq C 3^{-k_{0} d}
$$

with a constant $C>0$ independent of $k_{0}$, where $\mathbb{K}$ is a compact subset of $\mathbb{R}$, is called the approximation order of the scheme $\left\{S_{\boldsymbol{a}^{k}}\right\}_{k \geq 0}$.

Definition 5.8. 6] A non-stationary subdivision scheme $\left\{S_{\boldsymbol{a}^{k}}\right\}_{k \geq 0}$ is said to be asymptotically similar to a stationary subdivision scheme $S_{\boldsymbol{a}}$ if the masks $\left\{\boldsymbol{a}^{k}\right\}_{k \geq 0}$ and $\{\boldsymbol{a}\}$ have the same support $\mathcal{D}$ (i.e., $a_{i}^{k}=a_{i}=0$ for $i \notin \mathcal{D}$ ) and satisfy

$$
\lim _{k \rightarrow \infty} a_{\boldsymbol{\alpha}}^{k}=a_{\boldsymbol{\alpha}}, \quad \boldsymbol{\alpha} \in \mathcal{D} .
$$

Theorem 5.9. [8] Assume a non-stationary ternary scheme $\left\{S_{\boldsymbol{a}^{k}}\right\}_{k \geq 0}$ is $V_{T^{-}}$-reproducing and is asymptotically similar to a convergent ternary stationary scheme $\left\{S_{a}\right\}$. Assume 
further that the initial data are of the form $f^{d}:=\left\{f_{i}^{d}=f\left(3^{-d} i\right), i \in \mathbb{Z}\right\}$ for some fixed $d \geq 0$ and for some function $f \in W_{\infty}^{\gamma}(\mathbb{R})$ where $\gamma \in \mathbb{N}, \gamma \leq T$. If the Wronskian matrix $\mathrm{W}_{V_{\gamma}}(0)$ of $V_{\gamma} \subseteq V_{T}$ is invertible, where the $T \times T$ Wronskian matrix is defined by

$$
\mathrm{W}_{V_{T}}(x):=\left(\frac{1}{\beta !} \frac{d^{\beta} \varphi_{\alpha}(x)}{d x^{\beta}}, \alpha, \beta=0, \ldots, T-1\right)
$$

then

$$
\left\|g_{f^{d}}-f\right\|_{L_{\infty}(\mathbb{R})} \leq C_{f} 3^{-\gamma d}, \quad d \geq 0
$$

with a constant $C_{f}>0$ depending only on $f$, where $g_{f^{d}}$ is the limit of the subdivision scheme obtained from the initial data $f^{d}$.

In the following corollary, we give the approximation order of the non-stationary combined ternary subdivision scheme 4.1 by Theorem 5.9 .

Corollary 5.10. The non-stationary ternary combined subdivision scheme (4.1) has approximation order 4 when reproducing the space $\mathrm{EP}_{\Gamma_{1}, \Lambda_{1}}$, and the approximation order is 5 when reproducing the space $\mathrm{EP}_{\Gamma_{2}, \Lambda_{1}}$.

Compared to the scheme in 16, the approximation order of the new scheme (4.1) in the interpolating case is increased by 1 . Table 5.1 shows the maximal smoothness order, generation, reproduction and approximation order of the non-stationary ternary interpolating 5-point subdivision scheme in 16 and the new non-stationary combined ternary 5-point subdivision scheme 4.1.

\begin{tabular}{ccccccc}
\hline $\begin{array}{c}\text { Subdivision } \\
\text { scheme }\end{array}$ & Type & Support & Continuity & Generation & Reproduction & $\begin{array}{c}\text { Approximation } \\
\text { order }\end{array}$ \\
\hline $\begin{array}{c}\text { The scheme in 16 } \\
\text { The combined }\end{array}$ & Interpolating & 7 & $C^{2}$ & $\mathrm{EP}_{\Gamma_{1}, \Lambda_{1}}$ & $\operatorname{EP}_{\Gamma_{1}, \Lambda_{1}}$ & 4 \\
scheme 4.1 & Interpolating & 7 & $C^{2}$ & $\mathrm{EP}_{\Gamma_{2}, \Lambda_{1}}$ & $\operatorname{EP}_{\Gamma_{2}, \Lambda_{1}}$ & 5 \\
$\begin{array}{c}\text { The combined } \\
\text { scheme 4.1 }\end{array}$ & Approximating & 7 & $C^{4}$ & $\operatorname{EP}_{\Gamma_{3}, \Lambda_{1}}$, & & \\
& & & & $\operatorname{EP}_{\Gamma_{1}, \Lambda_{2}}$, & $\{1, x\}$ & 2 \\
\hline $\operatorname{EP}_{\Gamma_{1}, \Lambda_{2}, \Gamma_{1}}$ & & \\
\hline
\end{tabular}

Table 5.1: Comparison of the non-stationary ternary schemes.

\section{Conclusion}

In this paper, by suitably using the variable displacements, we have presented a family of non-stationary combined ternary 5-point subdivision schemes based on the ternary generalized subdivision of order 4 , which can reach $C^{2}$ in the interpolating case, and $C^{4}$ in 
the approximating scheme with suitable parameters. Moreover we investigate its generation/reproduction property and the approximation order. Compared to [16], the new scheme in the interpolating case has better reproduction property and the approximation order is increased by one. In the future, we may focus on the construction of non-stationary ternary subdivision schemes, which have better properties, such as smaller support, higher order of smoothness and better generation/reproduction property, by suitably using the displacement based on the odd order ternary generalized subdivision scheme.

\section{Proof of Theorem 3.4}

We prove Theorem 3.4 by induction on $m$. For $m=3$,

$$
\begin{aligned}
\sum_{i=1}^{n} P_{i} N_{i, 3}(l, t)= & \sum_{i=1}^{n} P_{i}\left(\frac{1}{l} \int_{t-l}^{t} N_{i, 2}(l, s) d s\right) \\
=\sum_{i=1}^{n} P_{i} & \frac{1}{3 \times \frac{l}{3}}\left(\left(\int_{t-l / 3}^{t}+\int_{t-2 l / 3}^{t-l / 3}+\int_{t-l}^{t-2 l / 3}\right) N_{i, 2}(l, s) d s\right) \\
=\sum_{i=1}^{n} P_{i} & {\left[\frac{1}{\left(1+2 \cos \frac{\omega l}{3}\right)^{2}} N_{3 i, 3}(l / 3, t)+\frac{1}{1+2 \cos \frac{\omega l}{3}} N_{3 i+1,3}(l / 3, t)\right.} \\
& +\frac{2 \cos \frac{\omega l}{3}}{1+2 \cos \frac{\omega l}{3}} N_{3 i+2,3}(l / 3, t)+\frac{4 \cos ^{2} \frac{\omega l}{3}+4 \cos \frac{\omega l}{3}-1}{\left(1+2 \cos \frac{\omega l}{3}\right)^{2}} N_{3 i+3,3}(l / 3, t) \\
& +\frac{2 \cos \frac{\omega l}{3}}{1+2 \cos \frac{\omega l}{3}} N_{3 i+4,3}(l / 3, t)+\frac{1}{1+2 \cos \frac{\omega l}{3}} N_{3 i+5,3}(l / 3, t) \\
& \left.+\frac{1}{\left(1+2 \cos \frac{\omega l}{3}\right)^{2}} N_{3 i+6,3}(l / 3, t)\right]
\end{aligned}
$$

Since $N_{i, 3}(l / 3, t)=0$ for $i=3,4,5,6,3 n+3,3 n+4,3 n+5,3 n+6$ and $t \in[3 l,(n+1) l]$, we get

$$
\begin{aligned}
& \sum_{i=1}^{n} P_{i} N_{i, 3}(l, t) \\
& =\sum_{i=1}^{n-2}\left[\left(\frac{2 \cos \frac{\omega l}{3}}{1+2 \cos \frac{\omega l}{3}} P_{i}+\frac{1}{1+2 \cos \frac{\omega l}{3}} P_{i+1}\right) N_{3 i+4,3}(l / 3, t)\right. \\
& +\left(\frac{1}{1+2 \cos \frac{\omega l}{3}} P_{i}+\frac{2 \cos \frac{\omega l}{3}}{1+2 \cos \frac{\omega l}{3}} P_{i+1}\right) N_{3 i+5,3}(l / 3, t) \\
& \left.+\left(\frac{1}{\left(1+2 \cos \frac{\omega l}{3}\right)^{2}} P_{i}+\frac{4 \cos ^{2} \frac{\omega l}{3}+4 \cos \frac{\omega l}{3}-1}{\left(1+2 \cos \frac{\omega l}{3}\right)^{2}} P_{i+1}+\frac{1}{\left(1+2 \cos \frac{\omega l}{3}\right)^{2}} P_{i+2}\right) N_{3 i+6,3}(l / 3, t)\right] \\
& +\left(\frac{2 \cos \frac{\omega l}{3}}{1+2 \cos \frac{\omega l}{3}} P_{n-1}+\frac{1}{1+2 \cos \frac{\omega l}{3}} P_{n}\right) N_{3 n+1,3}(l / 3, t) \\
& +\left(\frac{1}{1+2 \cos \frac{\omega l}{3}} P_{n-1}+\frac{2 \cos \frac{\omega l}{3}}{1+2 \cos \frac{\omega l}{3}} P_{n}\right) N_{3 n+2,3}(l / 3, t)
\end{aligned}
$$


$=\sum_{i=2}^{3 n-3} P_{i}^{3} N_{i+5,3}(l / 3, t)$.

Thus (3.2) holds.

Assume that the conclusion holds for $m-1(m \geq 4)$, i.e.,

$$
\begin{aligned}
\sum_{i=1}^{n} P_{i} N_{i, m-1}(l, t) & =\sum_{i=2}^{3 n-2 m+5} P_{i}^{m-1} N_{i+2 m-3, m-1}(l / 3, t), \\
P_{i}^{m-1} & =\frac{P_{i}^{m-2}+P_{i+1}^{m-2}+P_{i+2}^{m-2}}{3} .
\end{aligned}
$$

For any $m$, in view of Definition 3.1 and 7.1 , we have

$$
\begin{aligned}
& \sum_{i=1}^{n} P_{i} N_{i, m}(l, t) \\
= & \sum_{i=1}^{n} P_{i}\left(\frac{1}{l} \int_{t-l}^{t} N_{i, m-1}(l, s) d s\right) \\
= & \frac{1}{3 \times \frac{l}{3}}\left(\int_{t-l / 3}^{t}+\int_{t-2 l / 3}^{t-l / 3}+\int_{t-l}^{t-2 l / 3}\right)\left(\sum_{i=2}^{3 n-2 m+5} P_{i}^{m-1} N_{i+2 m-3, m-1}(l / 3, s) d s\right) \\
= & \frac{1}{3}\left[\sum_{i=2}^{3 n-2 m+5} P_{i}^{m-1} N_{i+2 m-3, m}(l / 3, t)+\sum_{i=2}^{3 n-2 m+5} P_{i}^{m-1} N_{i+2 m-2, m}(l / 3, t)\right. \\
& \left.+\sum_{i=2}^{3 n-2 m+5} P_{i}^{m-1} N_{i+2 m-1, m}(l / 3, t)\right] .
\end{aligned}
$$

Due to $N_{2 m-1, m}(l / 3, t)=N_{2 m, m}(l / 3, t)=N_{3 n+3, m}(l / 3, t)=N_{3 n+4, m}(l / 3, t)=0$ for $t \in$ $[m l,(n+1) l]$, after dropping terms involving these basis functions and rearranging the remaining terms in $(7.2)$, we arrive at

$$
\begin{aligned}
& \sum_{i=1}^{n} P_{i} N_{i, m}(l, t) \\
= & \frac{1}{3}\left[\left(P_{2}^{m-1}+P_{3}^{m-1}+P_{4}^{m-1}\right) N_{2 m+1, m}(l / 3, t)+\left(P_{3}^{m-1}+P_{4}^{m-1}+P_{5}^{m-1}\right) N_{2 m+2, m}(l / 3, t)\right. \\
& \left.\quad+\cdots+\left(P_{3 n-2 m+3}^{m-1}+P_{3 n-2 m+4}^{m-1}+P_{3 n-2 m+5}^{m-1}\right) N_{2 m+1, m}(l / 3, t)\right] \\
= & P_{2}^{m} N_{2 m+1, m}(l / 3, t)+P_{3}^{m} N_{2 m+2, m}(l / 3, t)+\cdots+P_{3 n+3-2 m}^{m} N_{3 n+2, m}(l / 3, t) \\
= & \sum_{i=2}^{3 n-2 m+3} P_{i}^{m} N_{i+2 m-1, m}(l / 3, t) .
\end{aligned}
$$

This proves the theorem. 


\section{References}

[1] C. Beccari, G. Casciola and L. Romani, Shape controlled interpolatory ternary subdivision, Appl. Math. Comput. 215 (2009), no. 3, 916-927.

[2] D. Burkhart, B. Hamann and G. Umlauf, Iso-geometric finite element analysis based on Catmull-Clark: subdivision solids, Comput. Graph. Forum 29 (2010), no. 5, 15751584.

[3] M. Charina, C. Conti, N. Guglielmi and V. Protasov, Limits of level and parameter dependent subdivision schemes: A matrix approach, Appl. Math. Comput. 272 (2016), part 1, 20-27.

[4] M. Charina, C. Conti and L. Romani, Reproduction of exponential polynomials by multivariate non-stationary subdivision schemes with a general dilation matrix, Numer. Math. 127 (2014), no. 2, 223-254.

[5] S. W. Choi, B.-G. Lee, Y. J. Lee and J. Yoon, Stationary subdivision schemes reproducing polynomials, Comput. Aided Geom. Design 23 (2006), no. 4, 351-360.

[6] C. Conti, N. Dyn, C. Manni and M.-L. Mazure, Convergence of univariate nonstationary subdivision schemes via asymptotic similarity, Comput. Aided Geom. Design 37 (2015), 1-8.

[7] C. Conti, L. Gori and F. Pitolli, Totally positive functions through nonstationary subdivision schemes, J. Comput. Appl. Math. 200 (2007), no. 1, 255-265.

[8] C. Conti, L. Romani and J. Yoon, Approximation order and approximate sum rules in subdivision, J. Approx. Theory 207 (2016), 380-401.

[9] M. Fang, W. Ma and G. Wang, A generalized curve subdivision scheme of arbitrary order with a tension parameter, Comput. Aided Geom. Design 27 (2010), no. 9, 720733.

[10] L. Gori and F. Pitolli, Nonstationary subdivision schemes and totally positive refinable functions, in: Approximation Theory XII, San Antonio 2007, 169-170, Mod. Methods Math., Nashboro Press, Brentwood, TN, 2008.

[11] M. F. Hassan and N. A. Dodgson, Ternary and three-point univariate subdivision schemes, in: Curve and Surface Fitting (Saint-Malo, 2002), 199-208, Mod. Methods Math., Nashboro Press, Brentwood, TN, 2003. 
[12] M. F. Hassan, I. P. Ivrissimitzis, N. A. Dodgson and M. A. Sabin, An interpolating 4-point $C^{2}$ ternary stationary subdivision scheme, Comput. Aided Geom. Design 19 (2002), no. 1, 1-18.

[13] A. Levin, Interpolating nets of curves by smooth subdivision surfaces, Proceedings of the 26th annual conference on Computer graphics and interactive techniques, (1999), $57-64$.

[14] J. Maillot and J. Stam, A unified subdivision scheme for polygonal modeling, Comput. Graph. Forum 20 (2001), no. 3, 471-479.

[15] P. Novara and L. Romani, Complete characterization of the regions of $C^{2}$ and $C^{3}$ convergence of combined ternary 4-point subdivision schemes, Appl. Math. Lett. 62 (2016), 84-91.

[16] _ On the interpolating 5-point ternary subdivision scheme: A revised proof of convexity-preservation and an application-oriented extension, Math. Comput. Simulation 147 (2018), 194-209.

[17] J. Pan, S. Lin and X. Luo, A combined approximating and interpolating subdivision scheme with $C^{2}$ continuity, Appl. Math. Lett. 25 (2012), no. 12, 2140-2146.

[18] K. Rehan and M. A. Sabri, A combined ternary 4-point subdivision scheme, Appl. Math. Comput. 276 (2016), 278-283.

[19] K. Rehan and S. S. Siddiqi, A family of ternary subdivision schemes for curves, Appl. Math. Comput. 270 (2015), 114-123.

[20] _ A combined binary 6-point subdivision scheme, Appl. Math. Comput. 270 (2015), 130-135.

[21] L. Romani, From approximating subdivision schemes for exponential splines to highperformance interpolating algorithms, J. Comput. Appl. Math. 224 (2009), no. 1, $383-396$.

[22] S. S. Siddiqi and K. Rehan, Modified form of binary and ternary 3-point subdivision schemes, Appl. Math. Comput. 216 (2010), no. 3, 970-982.

[23] L. Zhang, H. Ma, S. Tang and J. Tan, A combined approximating and interpolating ternary 4-point subdivision scheme, J. Comput. Appl. Math. 349 (2019), 563-578.

[24] H. Zheng, M. Hu and G. Peng, Constructing $2 n-1$ point ternary interpolatory subdivision schemes by using variation of constants, 2009 International Conference on Computational Intelligence and Software Engineering, (2009), 1-4. 
[25] H. Zheng and B. Zhang, A non-stationary combined subdivision scheme generating exponential polynomials, Appl. Math. Comput. 313 (2017), 209-221.

Zeze Zhang, Hongchan Zheng, Weijie Song and Baoxing Zhang

School of Mathematics and Statistics, Northwestern Polytechnical University, Xi'an, Shaanxi 710072, China

E-mail address: zhangzz@mail.nwpu.edu.cn, zhenghc@nwpu.edu.cn, veidge.son@nwpu.edu.cn, baoxingzhang@yeah.net 